\title{
A molecular dynamics study of structural transitions in small water clusters in the presence of an external electric field
}

\author{
Alice Vegiria) \\ National Hellenic Research Foundation, Institute of Theoretical and Physical Chemistry, \\ 48 Vas. Constantinou Avenue, Athens, 11635 Greece \\ Sergei V. Schevkunov \\ Physics and Mechanics Department, St. Petersburg State Technical University, 29 Politekhnicheskaya ul., \\ St. Petersburg, 195251 Russia
}

(Received 11 April 2001; accepted 6 June 2001)

\begin{abstract}
The present work constitutes a thorough study of the response of a relatively small water cluster $(N=32)$ to external static electric fields in the $0.5 \times 10^{7}$ to $10^{8} \mathrm{~V} / \mathrm{cm}$ range, at $T=200 \mathrm{~K}$. As the electric field is varied, the system undergoes a phase transition to structures resembling incomplete nanotubes consisting of stacked squares arranged perpendicularly to the field direction. For further field increase the system transforms continuously to more open structures, reminiscent of the proton ordered forms of cubic ice, found also in the liquid. Regarding the dynamic response of the cluster, this is reflected in a profound way on the nonmonotonic variation of the reorientational decay rates of the molecular intrinsic axes and of the self-diffusion coefficients along and perpendicular to the field lines. In general the external field induces a considerable increase of the reorientational decay rates of all axes, except for the strongest field where the electrofreezing effect is observed. Reorientational relaxation has been found to obey a stretched exponential behavior of the Kohlrausch-Williams-Watts-type, where a one-to-one correspondence between the $\beta$-exponent variation with the field, molecular cooperativity, and translational diffusion has been established. (C) 2001 American Institute of Physics. [DOI: 10.1063/1.1388545]
\end{abstract}

\section{INTRODUCTION}

Water becomes a more reactive environment when the extent of hydrogen bonding is reduced. A more hydrogenbonded network structure slows reactions due to its increased viscosity, reduced diffusivity, and the less active participation of water molecules. The application of external load (pressure), ${ }^{1-9}$ of electric field, ${ }^{10-24}$ of ultrasound flow, ${ }^{25}$ or the confinement of water thin films between plates or within cylindrical pores ${ }^{26,27}$ results in the break-up of the hydrogenbonding network and under certain conditions, the induction of a phase transition between different water forms. These effects remain largely unexplored despite the significance this knowledge has for understanding the solvation behavior and properties of water in biological systems.

The action of an electric field is through the torque it exerts to a water dipole in an attempt to reorient it along the field direction, thereby resulting in the perturbation of the molecular positions and the distortion or break-up of the hydrogen-bonding network. The induced dipole moments as well, by strong enough electric fields, can significantly alter the characteristics of the water-water interactions. Molecular dynamics (MD) studies, due to the limited simulation time, have been unable to produce crystalline forms of water by simply cooling down the liquid, since it becomes trapped in glassy metastable states. Electrofreezing of supercooled bulk water has become possible in simulations through the assistance of a strong electric field by Kusalik et al., ${ }^{11-14}$ where

${ }^{a)}$ Electronic mail: avegiri@eie.gr highly polarized cubic ice forms or a new quartz-like polymorph of ice, termed ice XII, ${ }^{14}$ have been obtained. The resultant crystalline structure at $T=200 \mathrm{~K}$ and for a field strength $E=5 \times 10^{7} \mathrm{~V} / \mathrm{cm}$ has been found to be mechanically stable even after the removal of the external field. In a subsequent work, the same authors extended their initial study to examine several other state points (temperatures and densities of the bulk), ${ }^{13}$ where it was concluded that field-induced crystallization can take place only within a limited range of densities, $\left(0.94-0.96 \mathrm{~g} / \mathrm{cm}^{3}\right)$ and certainly not for temperatures $T \geqslant 260 \mathrm{~K}$, even for very strong external fields. The formation of metastable cubic rather than of hexagonal ice, which is the usual ice form met in nature, is due to the diamond-like packing of cubic ice, which can support an ideal parallel alignment of the dipoles.

The application of an external field to bulk water under ambient conditions by Heinzinger et al. ${ }^{15}$ did not produce any observable structural transformation, except for the strongest of the fields $\left(10^{8} \mathrm{~V} / \mathrm{cm}\right)$, where a long-range order in the oxygen-oxygen radial distribution functions was detected. No tendency for crystallization, but an overall enhancement of the hydrogen-bonding network has been reported.

A recent simulation on liquid water by Jung $e t a .^{21}$ at $T=243 \mathrm{~K}$ reported an enhancement of structure regularity analogous to cubic ice under the application of an electric field in the 1.5 to $2 \times 10^{7} \mathrm{~V} / \mathrm{cm}$ range, whereas at a higher temperature, $T=263 \mathrm{~K}$, a larger field of $5 \times 10^{7} \mathrm{~V} / \mathrm{cm}$ was 
required for the observation of a structural change. At room temperature no changes have been recorded.

Yeh et al. ${ }^{17}$ have also performed simulations of bulk water under external fields of 5- and $10 \times 10^{7} \mathrm{~V} / \mathrm{cm}$, where a transition to an imperfect proton-ordered cubic ice has been observed only under the strongest field.

Computer simulations of water between conducting plates have reported a phase transition at a field strength of about $8 \times 10^{7} \mathrm{~V} / \mathrm{cm}$, which resulted in the destruction of the middle water layers at the phase transition point and the reconstruction of a new type of configuration which was distinguished predominantly by an interlayer, parallel to the field hydrogen-bonding network. ${ }^{22}$

A similar study by Zhu et al. ${ }^{19}$ concerning dynamical and structural features of confined water between charged plates, reported the break-up of the hydrogen-bond structure normal to the field as a result of the dipole alignment.

A restructuring of room temperature water towards a cubic ice-I form, near charged metal surfaces, has been observed by Watts in Ref. 18 under certain charge densities. With field increase, the crystallization of a larger domain, but not of all of the confined water, towards a strained cubic ice-I has been facilitated. The authors reported no substantial damage to the hydrogen-bonding network, which had been allocated to the system's ability to adapt to the new conditions by restructuring.

Electric field effects on confined Stockmayer fluids between charged plates ${ }^{20,24}$ have also been examined where a pronounced layering (the more pronounced, the lower the temperature $)^{24}$ of the particles between the plates has been recorded.

All these studies leave no doubt that water, either bulk or confined, can crystallize to cubic ice forms at low enough temperatures $(T \sim 250 \mathrm{~K})$ and for fields in the $2-10$ $\times 10^{7} \mathrm{~V} / \mathrm{cm}$ range. The strength of these fields is comparable to the average value of the local internal field in condensed water. However, most of these studies have been conducted at scattered electric field values and in a nonsystematic way, regarding the field dependence of the structural transformations that are taking place, the changes imposed on the hydrogen-bonding network, and the evolution of the dynamical properties (transport and orientational) of water molecules.

The present work is a continuation of a previous Monte Carlo study ${ }^{28}$ where we had examined the structures and the dependence of static properties such as melting temperature, susceptibility, and heat capacity of a water cluster, on an external uniform electric field, for temperatures in the 90$300 \mathrm{~K}$ range. We had found that electric fields of the order of $10^{7} \mathrm{~V} / \mathrm{cm}$ do not considerably affect the melting temperature $\left(T_{m} \sim 200 \mathrm{~K}\right)$ of a moderate in size cluster $(N=64)$, but they do enhance the molecular reorientational motions. In the present work we change the angle of observation by sticking to a particular temperature and by varying the strength of the electric field instead. Apart from a structural analysis, we mainly concentrate on the dynamical response of the molecules under the influence of a wide range of fields. The organization of the rest of the paper is as follows. In Sec. II the details of calculation are presented. In Secs. III and IV we examine the structural and dynamical properties, respectively, and Sec. V summarizes and concludes.

\section{METHOD OF CALCULATION}

We have carried out constant energy molecular dynamics (MD) simulations for a relatively small water cluster $(N$ $=32$ ) under several external electric fields in the $0-10$ $\times 10^{7} \mathrm{~V} / \mathrm{cm}$ range. The coupled Newton-Euler equations of motion for rigid bodies are integrated in time. To avoid singularities inherent to the Euler angle representation of the reorientations of rigid bodies, we adopted the quaternion formalism. ${ }^{29,30}$ As an integrator we used a variable time step, and variable order predictor-corrector algorithm as described by Shampine and Gordon. ${ }^{31}$ The accuracy of the integration was checked by the conservation of total energy and linear momentum.

At zero field, a high-energy initial configuration is slowly cooled down through five consecutive cycles to a temperature of about $200 \mathrm{~K}$, by appropriately scaling the translational and rotational velocities of the particles. The trajectory is further equilibrated at this temperature for about $0.5 \mathrm{~ns}$ and averages are collected for another $0.5 \mathrm{~ns}$. For subsequent runs under a different electric field, the last configuration of the previous run serves as the starting configuration for the next one.

The total energy of the system consists of the following terms:

$$
U=U_{\text {pair }}^{w-w}+U^{w-\text { field }}+U_{\text {pol }}^{w-\text { field }}+U_{\text {ind }}^{w-w},
$$

where $U_{\text {pair }}^{w-w}$ accounts for the pairwise water-water interactions. For this purpose the TIP4 $\mathrm{P}^{32}$ potential model has been employed, which has been found to adequately describe many liquid water and ice properties not only at ambient conditions but also in confinement, ${ }^{26,27}$ at increased pressure, ${ }^{1,3-5}$ at supercooled, ${ }^{11,33}$ or supercritical conditions. ${ }^{34,35}$ The TIP4P model is adequate for the description of small cluster properties as well, ${ }^{36,37}$ and is more susceptible to nucleation than the SPC/E potential. ${ }^{13}$

$U^{w-\text { field }}$ is the interaction energy between the permanent charges of water molecules and the external field, and is equal to

$$
U^{w-\text { field }}=-\sum_{i=1}^{N} \sum_{k=1}^{3} q_{k}\left(\mathbf{r}_{k}^{i} \cdot \mathbf{E}\right),
$$

where $\mathbf{r}_{k}^{i}$ is the position vector of the $k$ th point charge of the $i$ th molecule and $E$ is the external field directed along the $z$ axis.

$U_{\mathrm{pol}}^{w-\text { field }}$ is the polarization energy of water molecules under a uniform external field $E$. It is given as

$$
U_{\text {pol }}^{w-\text { field }}=-\alpha_{w} \frac{E^{2}}{2},
$$

where $a_{w}=1.44 \AA^{3}$ is the isotropic polarizability of an isolated water molecule.

$U_{\text {ind }}^{w-w}$ describes the induced dipole-permanent charge interactions between any two water molecules. 


$$
U_{\text {ind }}^{w-w}=-\sum_{i, j} \mathbf{E}_{i j}^{(1)} \cdot \mathbf{p}_{j}^{\text {ind }},
$$

where $\mathbf{E}_{i j}^{(1)}$ is the electric field produced by the permanent charge $q_{k}$ of the $i$ th water molecule at the oxygen atom of the $j$ th molecule

$$
\mathbf{E}_{i j}^{(1)}=\sum_{k=1}^{3} \frac{q_{k}}{\left|\mathbf{r}_{0}^{j}-\mathbf{r}_{k}^{i}\right|^{3}} \cdot\left(\mathbf{r}_{0}^{j}-\mathbf{r}_{k}^{i}\right),
$$

where $\mathbf{r}_{0}^{j}$ is the position vector of the oxygen atom of the $j$ th molecule.

$$
p_{i}^{\text {ind }}=\alpha_{w} \mathbf{E}
$$

is the dipole moment of the $i$ th molecule induced by the external electric field $\mathbf{E}$.

In this calculation induced dipole-induced dipole interaction terms have been neglected. Although these terms have proven to be significant in cases where the source of the electric field in the cluster is an embedded ion, the strengths of the uniform electric fields used here are roughly an order of magnitude less than the electric fields produced by an ion at contact distances. As a result, the induced dipole moment is also an order of magnitude less and the induced dipoleinduced dipole energy is two orders of magnitude less. For example, if we consider two water molecules at a contact distance of about $3 \AA$ and an external uniform electric field of $10^{7} \mathrm{~V} / \mathrm{cm}$, then the induced dipole-induced dipole interaction would amount to about $8.5 \times 10^{-17} \mathrm{erg}$, which for $T$ $=200 \mathrm{~K}$ would be equal to $0.003 k_{B} T$. This is a small number at the background of other approximations in the model. Of course, simultaneous action of several such terms acting in the same direction could magnify this effect. However, our estimations in this example have been made at contact distances where a limited number of water molecules can be found simultaneously. At larger distances induced dipoleinduced dipole interactions fall off as $\sim 1 / r^{3}$. Correspondingly, if we take the case of a negative ion, the electric field produced at the same distance $(3 \AA)$ would amount to 16 $\times 10^{7} \mathrm{~V} / \mathrm{cm}$, and the induced dipole-induced dipole interaction would be equal to $2.2 \times 10^{-14} \mathrm{erg}$, or $0.79 k_{B} T$. Through these two examples, we have shown that induced dipoleinduced dipole interaction terms should be taken into account in the case of ions as the sources of the electric field, but they could be neglected for field strengths of the magnitude considered here.

Temperature is determined from the average kinetic energy of the cluster, for all fields it was found to fluctuate evenly about a mean value, indicating cluster equilibration. Temperatures and their mean square deviations are listed in Table I.

We note that in the water cluster-electric field system, total angular momentum is not a constant of motion, except from the $L_{z}$ component, which is conserved. Since at $t=0$ the cluster is given zero total angular momentum (by assuming zero angular and translational velocities), $L_{z}$ remains zero throughout the calculation, but not the $L_{x}$ and the $L_{y}$ components, whose time variation depends on the time variation of the projection of the total dipole moment of the cluster on the $y$-and $x$ axes, respectively. The implications of a
TABLE I. Cluster temperature for different electric fields.

\begin{tabular}{cc}
\hline \hline$E$ & \\
$10^{7} \mathrm{~V} / \mathrm{cm}$ & $T / \mathrm{K}$ \\
\hline 0 & $192.4 \pm 11$ \\
0.5 & $190.9 \pm 11$ \\
1 & $201.2 \pm 12$ \\
1.5 & $210.7 \pm 14$ \\
2 & $202.6 \pm 12$ \\
3 & $205.4 \pm 12$ \\
4 & $216.8 \pm 13$ \\
6 & $208.8 \pm 11$ \\
10 & $206.8 \pm 10$ \\
\hline \hline
\end{tabular}

nonzero and nonconserved total angular momentum are that the translational and rotational motions of the individual water molecules are modulated by low frequency components, in the $3-10 \mathrm{~cm}^{-1}$ range depending on the field strength.

\section{STRUCTURE AND ORIENTATIONAL ORDERING}

It is now well established from calculations that the application of external fields of the order of $10^{8} \mathrm{~V} / \mathrm{cm}$ on liquid water results in the crystallization of the medium. Depending on density, temperature, and field strength, cubic ice forms have been obtained. By examining water clusters, because of their less demanding computational effort, we can get a more precise picture of the structural changes that are induced by the field, as well as the route followed towards crystallization. The dynamics of this transformation is examined in the following section.

In Fig. 1 we present oxygen-oxygen $g_{\text {oO }}(R)$ radial distribution functions (RDF) for several field strengths. It becomes immediately obvious that RDFs can be gathered into three distinct groups according to the field strength, implying in this way that as the field is varied to larger values at least two structural transitions are taking place, giving rise on the average to three different kinds of structures. Namely, the correlation functions that correspond to the weak-field regime $E=0-1.0 \times 10^{7} \mathrm{~V} / \mathrm{cm}$ constitute the first group. The structures that correspond to the intermediate field regime, $E=1.5-2.75 \times 10^{7} \mathrm{~V} / \mathrm{cm}$, give rise to a second distinct group

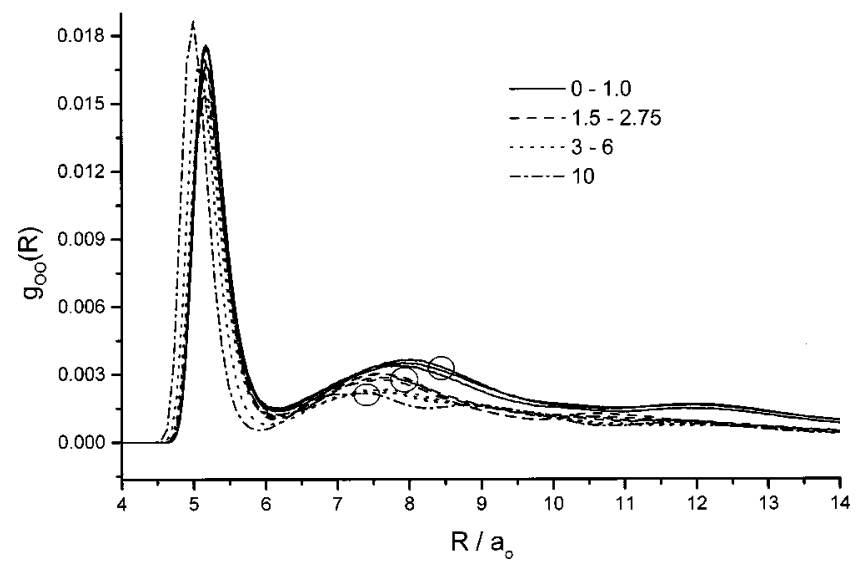

FIG. 1. Oxygen-oxygen radial distribution functions for different electric fields. $R$ is in atomic units and $E$ in $10^{7} \mathrm{~V} / \mathrm{cm}$. 


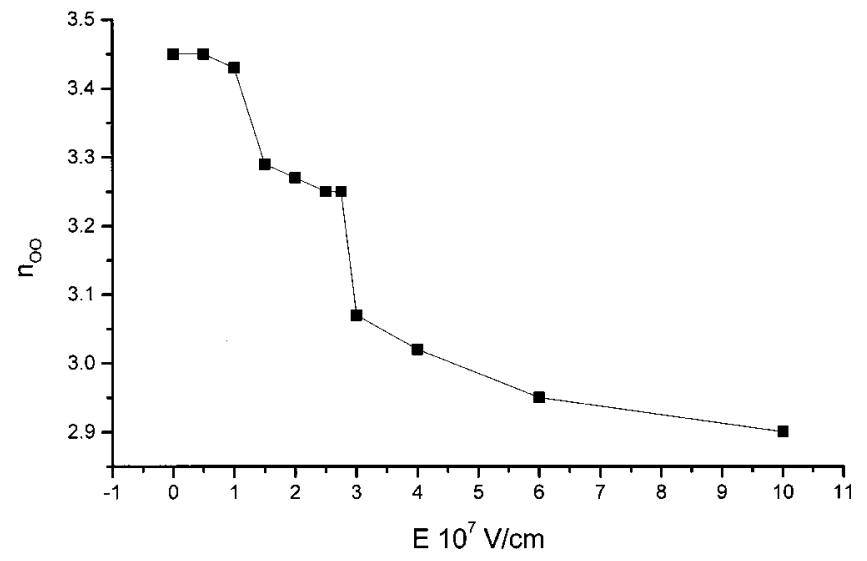

FIG. 2. Oxygen atom coordination number as a function of the external field.

of correlation functions, different from the first group, as it is manifested by the considerable approach of the second neighbors to smaller distances and the lessening of the second coordination shell intensities. Finally, the structures that correspond to fields from $E=3 \times 10^{7}-10^{8} \mathrm{~V} / \mathrm{cm}$ (strong field regime), constitute the third group, which is characterized by the further approach and intensity reduction of first-and second neighbors. Specifically, for $E=10 \times 10^{7} \mathrm{~V} / \mathrm{cm}$ the significant shift of the first coordination shell to 5.0ao (from about 5.2ao at $E=0)$ and the development of multiple wellshaped undulations that survive up to large distances signifies the presence of a quite ordered structure, different in form from those obtained at smaller fields.

The distinction into three individual groups of fieldinduced structural types is also reproduced in the variation of the oxygen atom coordination number. As shown in Fig. 2, each group is characterized by a similar coordination number, whereas for the third group this quantity decreases in a continuous fashion.

A similar distinction can be also applied to the distribution of the angles of the dipole moment vector with respect to the field direction. The results are presented in Fig. 3 as a function of the field strength. The distributions for $E$

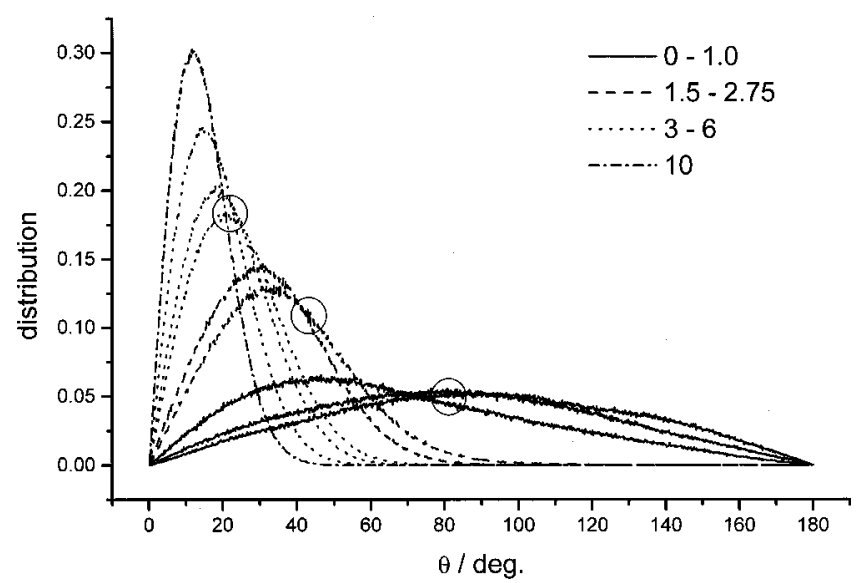

FIG. 3. Distributions of the angles between the dipole moment vector of a molecule and the direction of the external electric field, for various fields. $E$ is in $10^{7} \mathrm{~V} / \mathrm{cm}$.

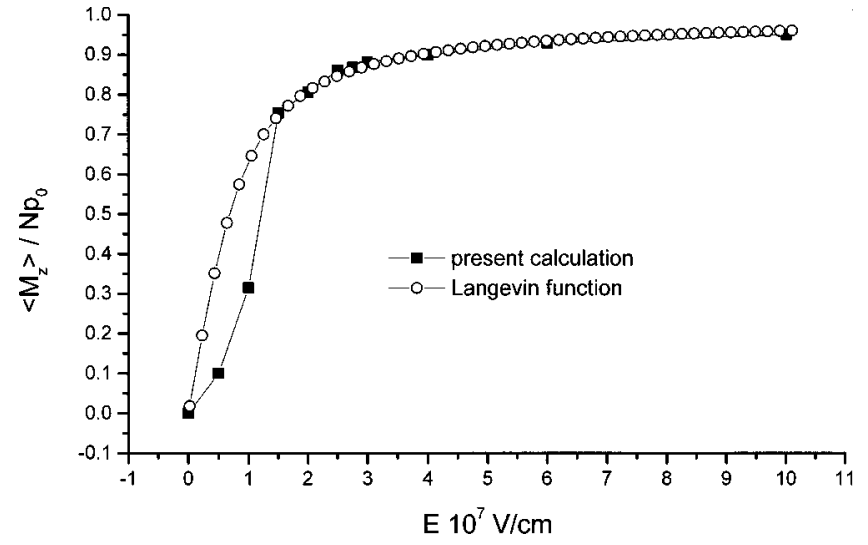

FIG. 4. Cluster polarization as a function of the external field.

$=0-1.0 \times 10^{7} \mathrm{~V} / \mathrm{cm}$ are nearly symmetric and broad, extending over all the $[0-\pi]$ angular range, implying a random-like distribution of the molecular orientations with respect to the $z$ axis. However, for $E=1.5 \times 10^{7} \mathrm{~V} / \mathrm{cm}$ an abrupt change in the shape of the distribution and in the value of the angle that corresponds to the maximum of this distribution is taking place, as if a sudden loss of the orientational randomness is taking place. It is characteristic that for fields $E \geqslant 1.5$ $\times 10^{7} \mathrm{~V} / \mathrm{cm}$ all distributions die off at angles larger than 90 deg, which implies that all dipoles are oriented within the upper half $[0-\pi / 2]$ angular hemisphere. For $E \geqslant 1.5$ $\times 10^{7} \mathrm{~V} / \mathrm{cm}$ the distributions become more asymmetric with their maximum intensities displaced to smaller angles and larger values, as the dipoles progressively acquire a betterdefined orientation.

The field impact on dipole alignment is indicated through the projection of the total dipole moment of the cluster on the field axis, $M_{z}$, which is plotted in Fig. 4 as a function of the field strength. It is surprising that for fields $E \geqslant 1.5 \times 10^{7} \mathrm{~V} / \mathrm{cm}$, the calculated $\left\langle M_{z}\right\rangle$ follows the Langevin equation exactly

$$
\left\langle M_{z}\right\rangle=A\left[\operatorname{cotanh}(\alpha E)-\frac{1}{\alpha E}\right],
$$

which has been derived according to Boltzmann statistics for a noninteracting dipolar gas.

$$
A=N p_{0} \quad \text { and } \quad \alpha=p_{0} / k_{B} T,
$$

which, for $N=32, T=200 \mathrm{~K}$, and $p_{0}=0.865$ a.u. (the dipole moment of a TIP4P water molecule), are equal to 27.68 and 1365.8 a.u., respectively.

This can be understood if we examine the field dependence of the average water-water, $U^{W-W}$ and water-field, $U^{W-E}$ interactions in the cluster, which are displayed in Fig. 5. For weak fields, the $U^{W-E}$ term is unimportant compared to $U^{W-W}$, therefore, the departure from the Langevin behavior is justified. The polarization of the cluster is smaller than that predicted by the Langevin equation, due to the attractive water-water interactions, which by acting as a drag on the fluctuations of the dipole moment vectors inhibit their net alignment. At $E=1.5 \times 10^{7} \mathrm{~V} / \mathrm{cm}$ we observe a jump in water-water interaction to smaller values as the result of the onset of the molecular dipole alignment and the subsequent 


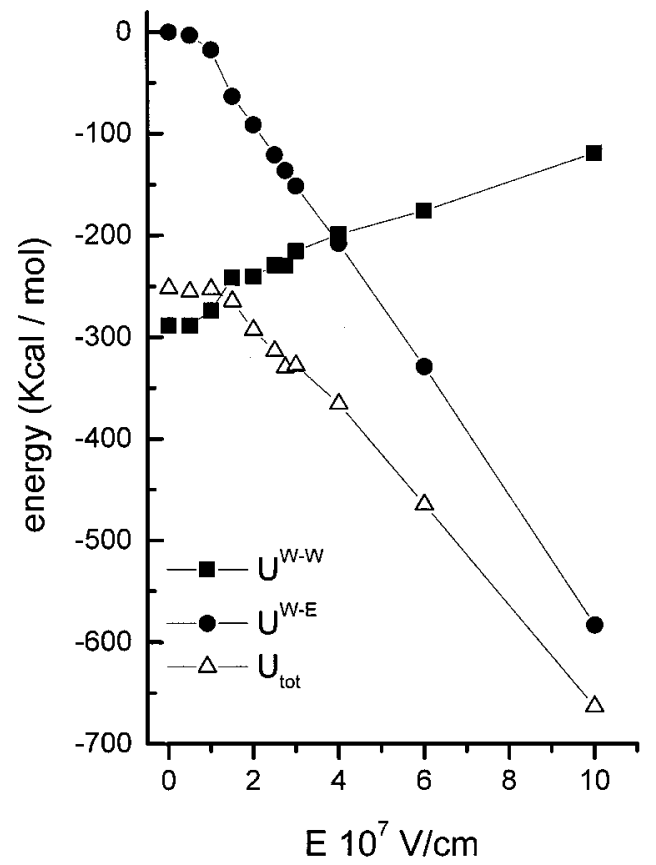

FIG. 5. Water-water $\left(U^{W-W}\right)$, water-external field $\left(U^{W-E}\right)$, and total energy $\left(U_{\text {tot }}\right)$ variation as a function of the electric field.

repulsion of neighboring dipoles. For stronger fields, the $U^{W-E}$ terms gain progressively in importance compared to the $U^{W-W}$ interactions and the Langevin behavior is established.

The fact that the discontinuities observed in the variation of the variables and distributions presented above are due to a structural phase transition is reflected in the total dipole moment fluctuations $\left(\left\langle M^{2}\right\rangle-\langle M\rangle^{2}\right)$ as a function of $E$, Fig. 6. Fluctuations become pronounced at $E=1.5 \times 10^{7} \mathrm{~V} / \mathrm{cm}$, leaving no doubt about the appearance of a new phase. Fluctuations at $3 \times 10^{7} \mathrm{~V} / \mathrm{cm}$, however, are within the statistical accuracy and cannot be taken as an indication of a phase transition.

Radial distribution functions over all space suit are better suited for the description of structures that are closer to a spherical shape, like the cluster at $E=0$. Since a cluster in the presence of a field is expected to depart from the spheri-

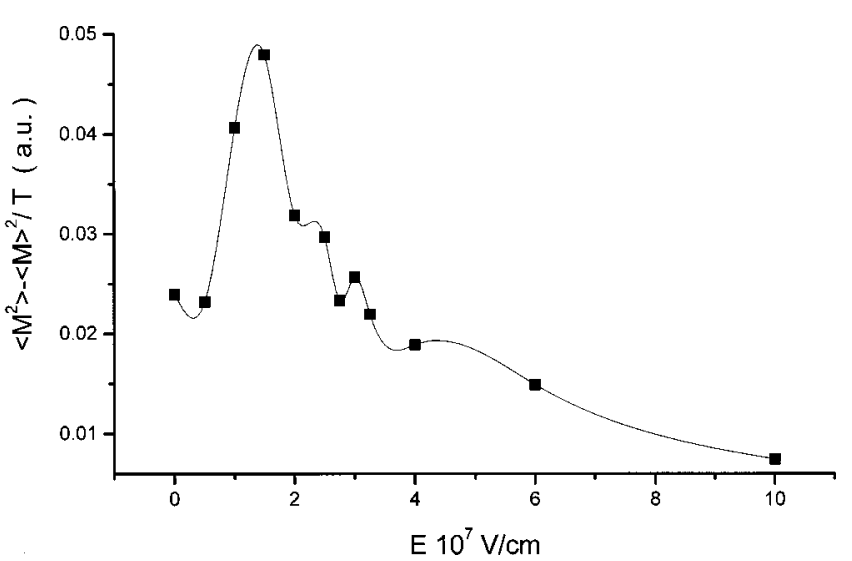

FIG. 6. Polarization fluctuations as a function of the electric field.

cal geometry due to elongation, we have chosen to present pair distributions of the water molecules (a) over $x-y$ planes perpendicular to the $z$ axis, and (b) over $x-z$ planes parallel to it. The corresponding horizontal, $g_{\mathrm{O}-\mathrm{O}}^{H}(R)$ and vertical, $g_{\mathrm{O}-\mathrm{O}}^{V}(R)$ oxygen atom radial distributions are displayed in Figs. 7(a) and (b) for $E=0,1,1.5,2,3,6$, and 10 $\times 10^{7} \mathrm{~V} / \mathrm{cm}$. The curves are normalized so that

$$
2 \pi \int g_{\mathrm{OO}}(R) R d R=1 .
$$

We have also made estimations of the volume of the cluster, through the computation of the cluster average density in an $x-y$ plane and along the $z$ axis. For all fields except $E=0$, where the cluster is taken as a sphere, the cluster is assumed to have a cylindrical shape.

Regarding $g_{\mathrm{O}-\mathrm{O}}^{H}(R)$, one of the most characteristic changes with the switching on of the field is the significant reduction of the intensity of the first shell to almost half of its value at $E=0$. The same behavior is observed for $g_{\mathrm{O}-\mathrm{O}}^{V}(R)$ as well, where the population of the second shell is also reduced. Thus, the electric field rearranges the neighborhood of a particular molecule so that more free space becomes available in its vicinity. Not only the immediate environment, but also the volume of the cluster as a whole is increasing as well.

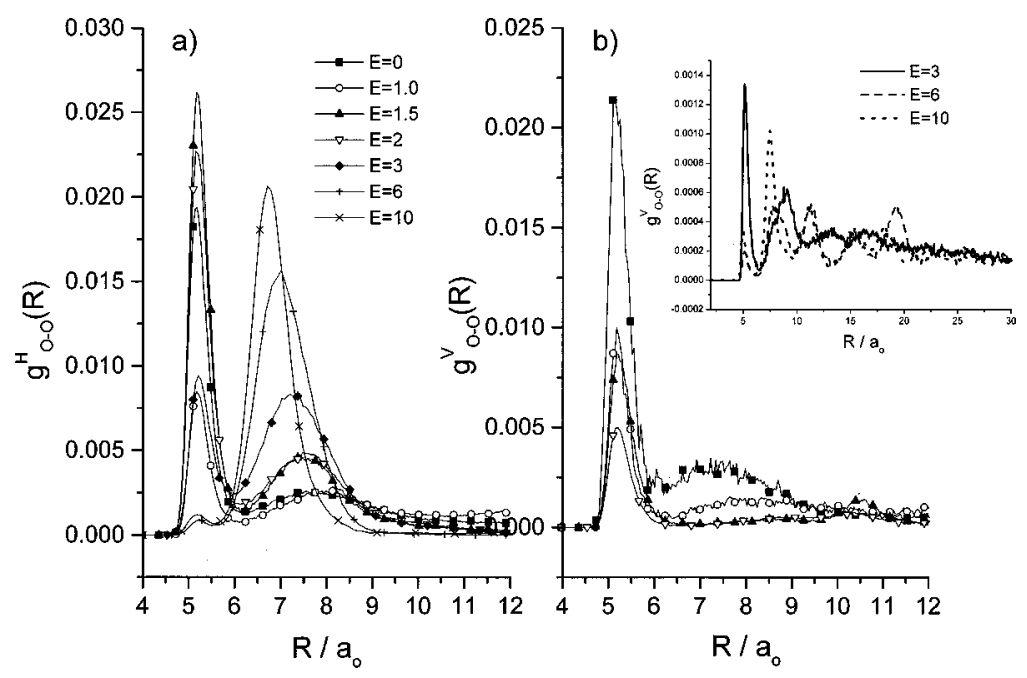

FIG. 7. (a) $g_{\mathrm{O}-\mathrm{O}}^{H}(R)$ and (b) $g_{\mathrm{O}-\mathrm{O}}^{V}(R) 2 \mathrm{D}$ correlation functions for various electric fields. $E$ is in $10^{7} \mathrm{~V} / \mathrm{cm}$. 
a)
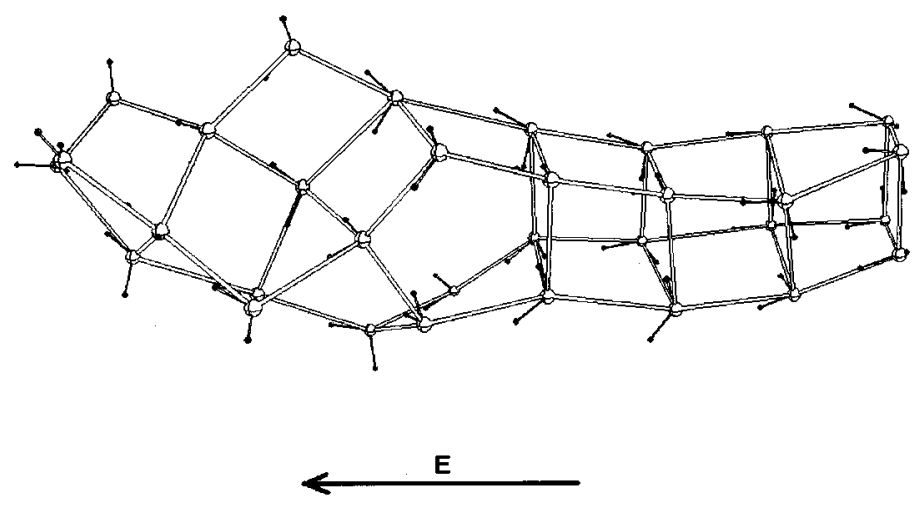

b)

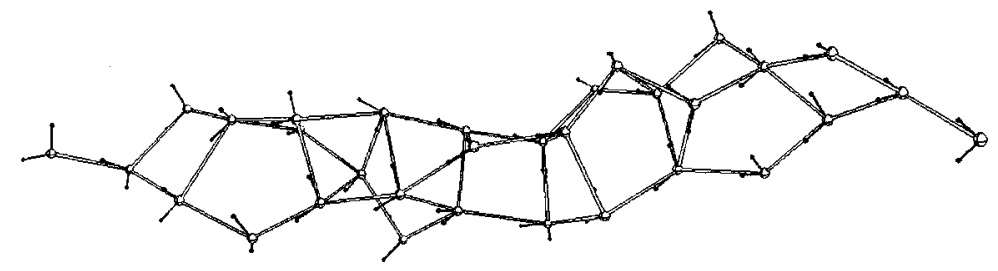

(c)

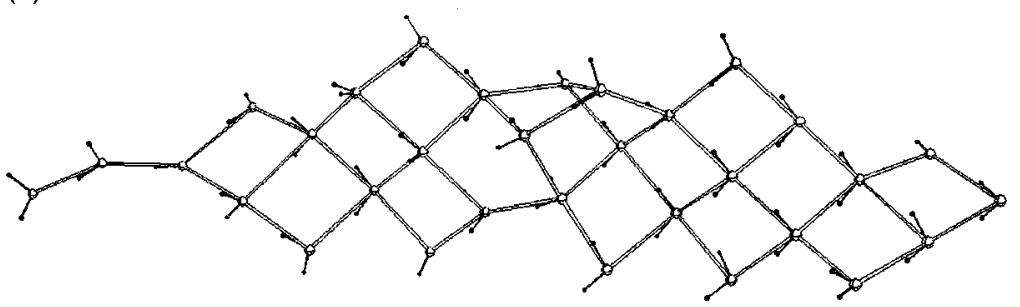

FIG. 8. Representative cluster configurations at (a) $E$ $=1.5 \times 10^{7} \mathrm{~V} / \mathrm{cm} ;$ (b) $E=3.0 \times 10^{7} \mathrm{~V} / \mathrm{cm} ;$ and (c) $E$ $=10^{8} \mathrm{~V} / \mathrm{cm}$.
In the intermediate field regime we observe a back transference of molecules into the first and second shells on the horizontal plane, but a continuing depletion in the vertical direction. At the same time, the cluster volume starts decreasing.

In the strong-field regime the most dramatic changes are observed in $g_{\mathrm{O}-\mathrm{O}}^{H}(R)$ with the transference of molecules to the second shell and the depletion of the first shell. Thus, while at $E=0$ the most probable separation of the water molecules in the $x-y$ plane is $5.2 \mathrm{ao}$; this distance increases to 6.7 ao for $E=10 \times 10^{7} \mathrm{~V} / \mathrm{cm}$. This can be understood as the energetic compromise of the cluster to reduce the repulsion of parallel water dipoles, fully aligned along the field. The build-up of a quasicrystalline phase with a pronounced long-range order in the cluster to directions along the field lines is clearly shown in the inset of Fig. 7(b). At the same time, cluster volume starts increasing again.

Which are, however, the new structures that emerge under the field application? By examining representative snapshots for $E=1.5,3$, and $10 \times 10^{7} \mathrm{~V} / \mathrm{cm}$, displayed in Fig. 8, we can associate the structural phase transition at $E=1.5$ $\times 10^{7} \mathrm{~V} / \mathrm{cm}$ with the partial nucleation of water molecules to square nanotubes produced by the stacking of two to four square rings at one edge of the cluster along the field direction. Although, ice nanotubes consisting of a small number of molecules have been found to be energetically more favorable when the rings are squares, ${ }^{38}$ the particular molecular arrangement we observe here is not of the lowest energy. Perhaps a narrow range of temperatures and fields may exist where nucleation to a complete square superlattice will become possible. The structures at $E=3 \times 10^{7} \mathrm{~V} / \mathrm{cm}$ correspond to the destruction of the nanotube phase, whereas those at $E=10^{8} \mathrm{~V} / \mathrm{cm}$ resemble those of the cubic ice, proton-ordered conformations found in bulk water upon electrofreezing.

From the evolution of the average cluster configuration as a function of the field, we can say that a sequence of field-induced transformations (random $\rightarrow$ partially ordered $\rightarrow$ random $\rightarrow$ ordered) is taking place. Specifically, weak fields $\left(E \leqslant 1.0 \times 10^{7} \mathrm{~V} / \mathrm{cm}\right)$ induce a weakening of the zerofield structure of the cluster. After a certain field value the cluster takes a quasicrystalline form (nanotubes), which survives for a limited range of field strengths $\left(1.5 \times 10^{7} \leqslant E\right.$ $\left.\leqslant 2.75 \times 10^{7} \mathrm{~V} / \mathrm{cm}\right)$. For larger fields this structure is destroyed, until at even larger ones $\left(E \geqslant 6 \times 10^{7} \mathrm{~V} / \mathrm{cm}\right)$ a new quasicrystalline phase emerges (cubic ice). Although the cubic crystalline phase has been routinely observed in simulations of supercooled bulk or 2D water confined between plates, the appearance of a nanotube high-energy phase is an attribute of the cluster alone and not of the bulk. 


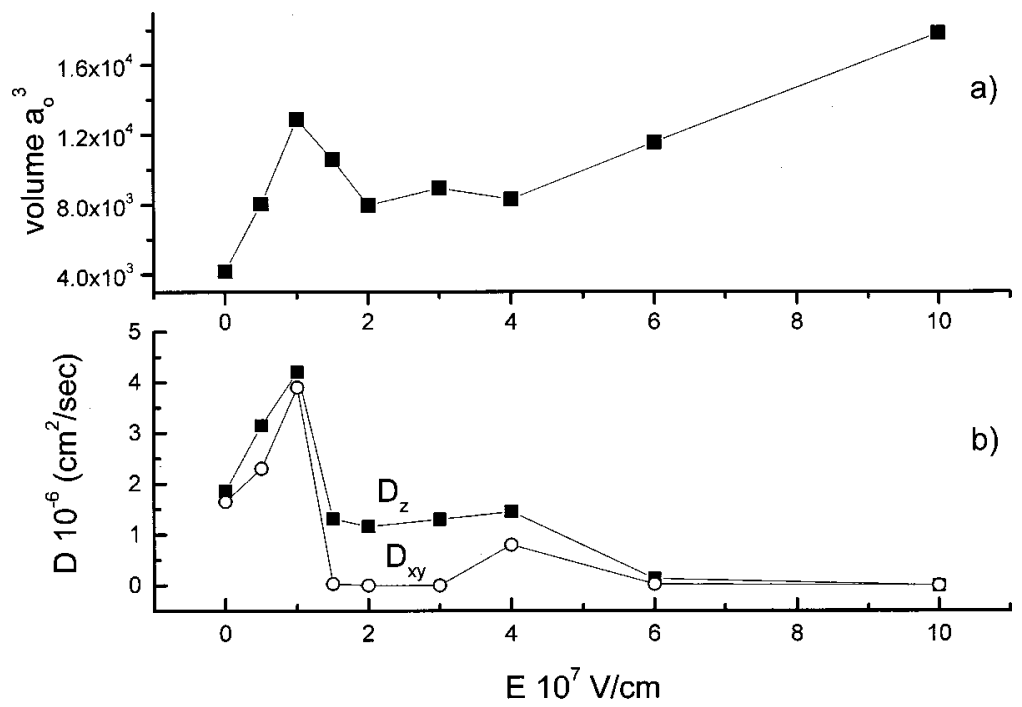

FIG. 9. (a) Cluster volume; (b) longitudinal and transverse self-diffusion coefficients.

b)

\section{DYNAMICS}

\section{A. Translational diffusion}

In this section we study the anisotropic diffusional motion of the molecules in directions along and perpendicular to the field, by means of transverse $\left(D_{x y}\right)$ and longitudinal $\left(D_{z}\right)$ self-diffusion coefficients, which are calculated from the long time slope of the appropriate mean square displacement (MSD) curves according to the Einstein relation

$$
D=\frac{1}{n_{t \rightarrow \infty}} \lim _{t} \frac{\left\langle\left|r_{i}(t)-r_{i}(0)\right|^{2}\right\rangle}{t} .
$$

Here, $n$ is equal to 2 and 4 for $D_{z}$ and $D_{x y}$, respectively.

Molecular diffusion shows a complicated dependence on field which cannot be explained independently of the structural transformations that are taking place in the cluster at the same time. On one hand we observe an anisotropic diffusion along and perpendicular to the field, and on the other, a nonmonotonic variation of the diffusion coefficient with the field. The most interesting effect, however, is that with the field switched on there is an increase of both $D_{z}$ and $D_{x y}$ diffusion coefficients, which take their maximum values at $E=1.0 \times 10^{7} \mathrm{~V} / \mathrm{cm}$. With further field increase they both decrease, with $D_{x y}$ faster than $D_{z}$.

Such a behavior resembles the nonmonotonic dependence of viscosity on pressure in liquid water. When pressure is applied to a simple liquid, there is an increase in viscosity and decrease in diffusion in a monotonic way. However, for liquid water below $300 \mathrm{~K}$ (Ref. 1) the initial application of pressure results in the increase of the diffusion constant. This effect has been attributed to the weakening of the hydrogenbonding network, which, however, is compensated for by the increase of density due to the external pressure. Further application of pressure to the liquid causes the diffusion coef-
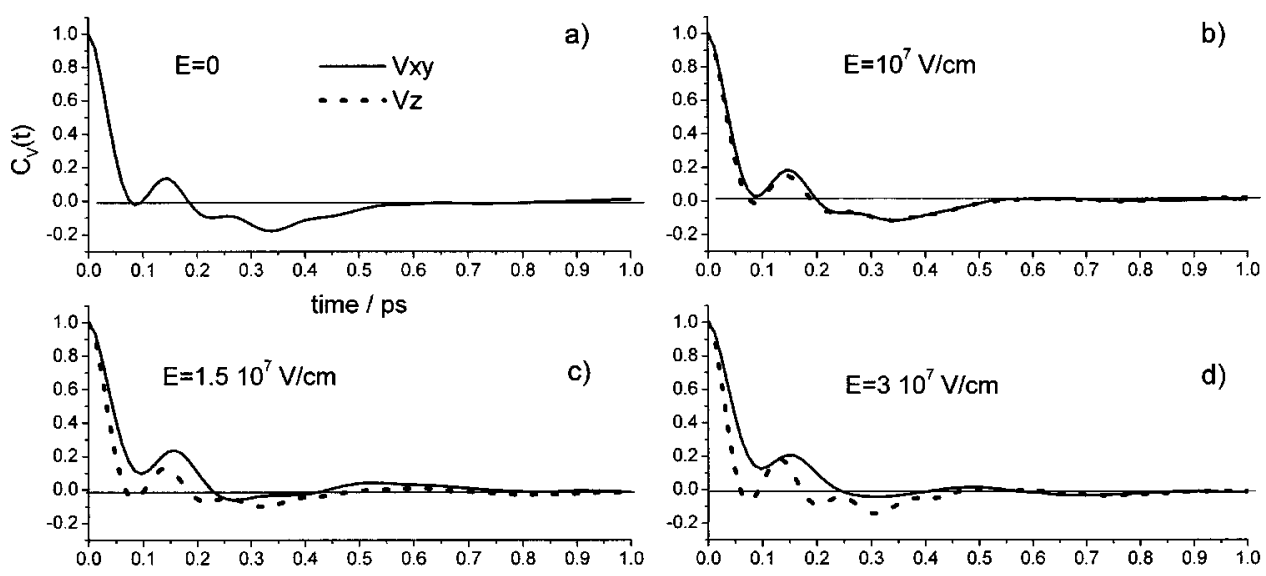

FIG. 10. Longitudinal and transverse velocity autocorrelation functions for various electric fields.
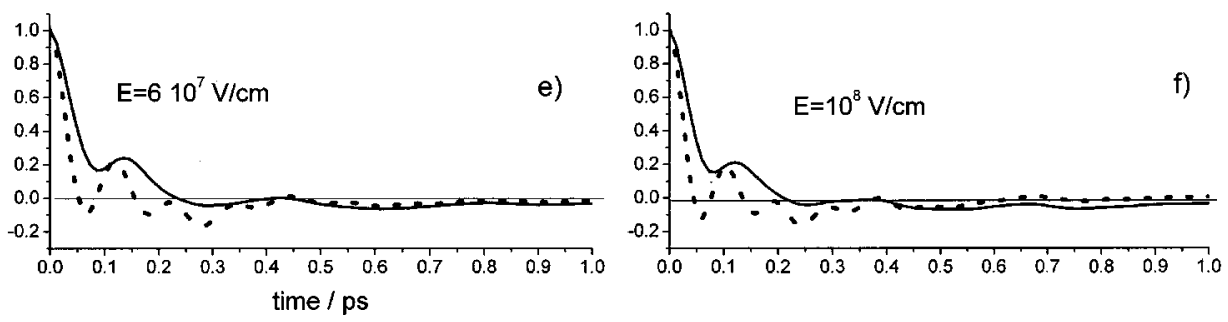

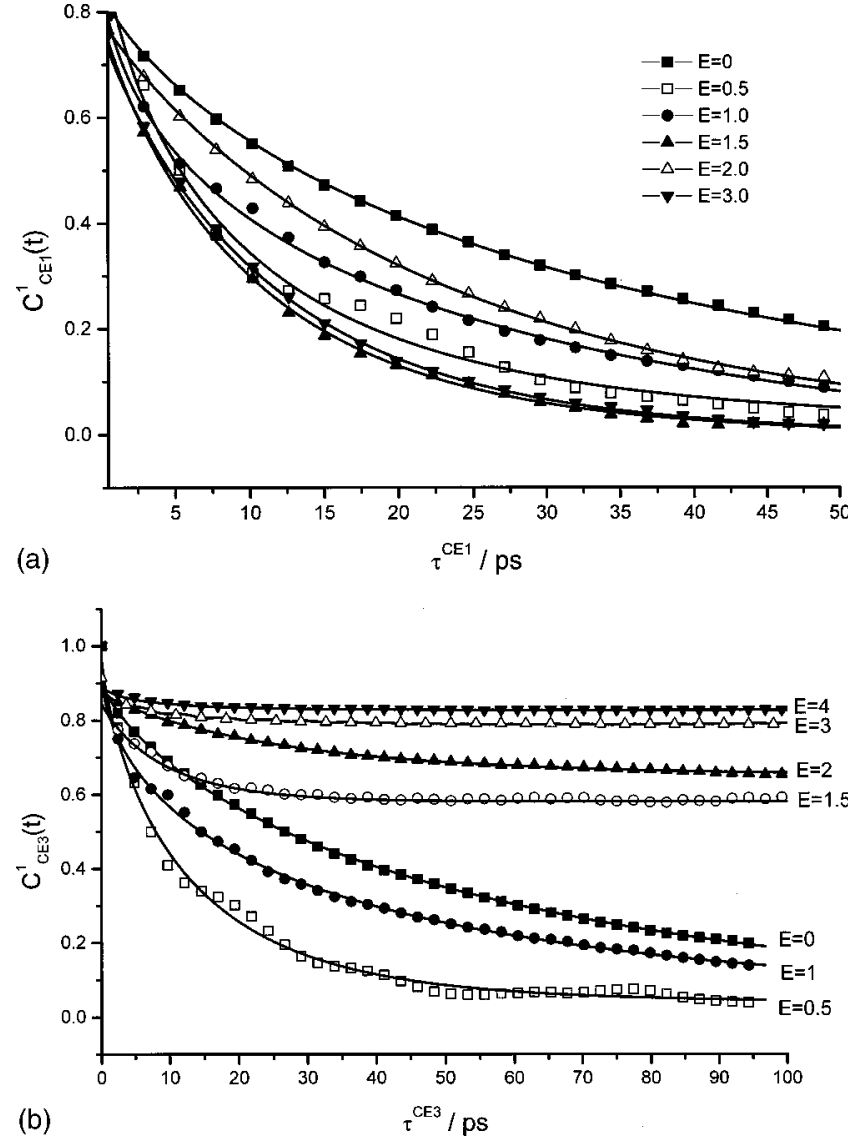

FIG. 11. (a) $C_{\mathrm{CE} 1}^{1}(t)$ and (b) $C_{\mathrm{CE} 3}^{1}(t)$ reorientational correlation functions for various fields. $E$ is in $10^{7} \mathrm{~V} / \mathrm{cm}$.

ficient to pass through a maximum and then decrease.

Concerning clusters, however, although $D_{x y, z}$ varies in a similar way with the external field, this behavior is very closely related to the variations of the volume of the cluster, which as we have already seen first increase for weak fields, then decrease for intermediate fields, and finally start increasing again for stronger fields. The close correlation of the diffusion constant to the variations of cluster volume is shown in Figs. 9(a) and (b). This correspondence breaks down for the stronger fields, where the decrease of diffusion is due to cluster crystallization.

Regarding the behavior of the self-diffusion coefficient under the influence of an external field in bulk water at ambient conditions, computer simulations with the SPC/E water model $\left(T=300 \mathrm{~K}\right.$ and $\left.E=5-20 \times 10^{7} \mathrm{~V} / \mathrm{cm}\right)$ have shown a monotonic decrease of $D$ with increasing field. ${ }^{15}$ However, no anisotropy between transverse and longitudinal displacements has been detected. In contrast, similar calculations for the TIP4P water model, at lower temperature, $T=243 \mathrm{~K}$ and $E=5 \times 10^{7} \mathrm{~V} / \mathrm{cm}$ in Ref. 21 , have shown a pronounced anisotropy, with the $D_{z}$ coefficients being almost double the magnitude of the $D_{x y}$ ones. However, no physical explanation for this effect has been given.

A microscopic view of the cluster dynamic behavior under different electric fields can also be obtained from the longitudinal $C_{V}^{z}(t)$, and transverse $C_{V}^{x y}(t)$, to the field components of the velocity autocorrelation functions (VAF) of the oxygen atoms, which are displayed in Figs. 10(a)-10(f). In the weak-field regime no anisotropy in VAF is observed. The curves show an extended negative plateau, which is to be expected in low temperature systems, due to the particle rebound motions from near neighbors. The anisotropy in motion becomes evident for fields $E \geqslant 1.5 \times 10^{7} \mathrm{~V} / \mathrm{cm}$, where the $C_{V}^{x y}(t)$ component almost loses its negative region because of the disruption of the horizontal hydrogen-bonding network. On the other hand, the $C_{V}^{z}(t)$ component develops additional oscillations, due to crystallization, which progressively shift to smaller times (larger frequencies). The observed frequency increase is consistent with the expected behavior of water with strained hydrogen bonds and distorted $\mathrm{O}-\mathrm{O}-\mathrm{O}$ angles. ${ }^{9}$ In fact, in the current case with the fieldinduced structural transformation of the cluster to forms consisting of either cubes or squares, the departure of the $\mathrm{O}-\mathrm{O}-\mathrm{O}$ angles from their tetrahedral values is to be expected.

\section{B. Axes reorientation}

In the following we examine the field effect on the reorientational relaxation of individual molecules. In this respect, we calculate the first-rank rotational autocorrelation function $C_{a}^{1}(t)$ for the three intrinsic molecular axes. We define as CE1, the axis that is perpendicular to the molecular plane, CE2 the axis that is along the HH direction, and CE3 the molecular symmetry axis that coincides with the direction of the dipole moment vector. Motion of the dipole axis is associated with the molecular tumbling motions, whereas motions around the CE1 and CE2 axes has to do with excitation of both tumbling and spinning molecular motions

$$
C_{a}^{1}(t)=\left\langle P_{1}\left[\hat{e}^{a}(\tau) \hat{e}^{a}(0)\right]\right\rangle,
$$

where $\hat{e}^{\alpha}$ is a unit vector along the corresponding $\alpha$ th axis (CE1, CE2, CE3) and $P_{1}$ is the first-order Legendre polynomial.

The limiting value of the correlation functions at long times is equal to

$$
\left\langle e_{x}^{\alpha}\right\rangle^{2}+\left\langle e_{y}^{\alpha}\right\rangle^{2}+\left\langle e_{z}^{\alpha}\right\rangle^{2},
$$

where

$$
\left\langle e_{x}^{\alpha}\right\rangle=\left\langle e_{y}^{\alpha}\right\rangle=\left\langle e_{z}^{\alpha}\right\rangle=0, \quad \text { for } \quad \alpha=\mathrm{CE} 1, \mathrm{CE} 2,
$$

and

$$
\left\langle e_{z}^{\alpha}\right\rangle=\left\langle M_{z}\right\rangle / N p_{0} \quad \text { for } \quad \alpha=\mathrm{CE} 3 .
$$

In the absence of electric field, $\left\langle M_{z}\right\rangle=0$.

The $C_{\mathrm{CE} 1}^{1}(t)$ and $C_{\mathrm{CE} 3}^{1}(t)$ autocorrelation functions as a function of the external field are displayed in Figs. 11(a) and 11(b). The orientational relaxation of the CE2 axis is the same as that of CE1 and is not displayed. We have found that the correlation functions are adequately represented not by a single but by a stretched exponential of the KohlrauschWilliams-Watts (KWW) type, ${ }^{39}$ shown in Fig. 11 as a continuous line.

$$
A \exp (-t / \tau)^{\beta}+\left(\left\langle P_{z}\right\rangle / N p_{0}\right)^{2} \quad \text { where } \quad<0<\beta \leqslant 1 .
$$

The deviation from the KWW function observed in particular for $E=0.5 \times 10^{7} \mathrm{~V} / \mathrm{cm}$ is due to the low frequency 
TABLE II. Stretched exponential fitting parameters of the $C_{\mathrm{CE} 1}^{1}(t), C_{\mathrm{CE} 2}^{1}(t)$, and $C_{\mathrm{CE} 3}^{1}(t)$ autocorrelation functions.

\begin{tabular}{|c|c|c|c|c|c|c|c|c|c|}
\hline \multirow[b]{2}{*}{$E=10^{7} \mathrm{~V} / \mathrm{cm}$} & \multicolumn{3}{|c|}{$C_{\mathrm{CE} 1}^{1}(t)$} & \multicolumn{3}{|c|}{$C_{\mathrm{CE} 2}^{1}(t)$} & \multicolumn{3}{|c|}{$C_{\mathrm{CE} 3}^{1}(t)$} \\
\hline & $A$ & $\tau / \mathrm{ps}$ & $\beta$ & $A$ & $\tau / \mathrm{ps}$ & $\beta$ & $A$ & $\tau / \mathrm{ps}$ & $\beta$ \\
\hline 0 & 0.85 & 30.4 & 0.77 & 0.87 & 40.1 & 0.91 & 0.91 & 53.1 & 0.75 \\
\hline 0.5 & 0.96 & 8.9 & 0.72 & 1.0 & 10.2 & 0.71 & 0.93 & 12.4 & 0.79 \\
\hline 1 & 1.0 & 19.9 & 0.56 & 1.02 & 19.7 & 0.63 & 0.91 & 30.7 & 0.61 \\
\hline 1.5 & 0.80 & 10.2 & 0.89 & 0.81 & 11.6 & 0.84 & 0.27 & 9.3 & 0.92 \\
\hline 2 & 0.79 & 22.3 & 0.93 & 0.81 & 25.4 & 0.86 & 0.22 & 26.3 & 0.91 \\
\hline 3 & 0.77 & 11.4 & 0.93 & 0.81 & 11.6 & 0.90 & 0.12 & 5.0 & 0.55 \\
\hline 4 & 0.73 & 10.2 & 0.95 & 0.76 & 10.9 & 0.94 & 0.077 & 6.1 & 0.66 \\
\hline 6 & 0.73 & 14.8 & 1.0 & 0.74 & 16.2 & 0.99 & 0.041 & 13.7 & 0.72 \\
\hline 10 & 0.84 & 50.6 & 0.91 & 0.84 & 49.5 & 1.0 & 0.026 & 37.8 & 0.88 \\
\hline
\end{tabular}

modulations of the corresponding correlation functions from the $x y$ components of the total angular momentum of the cluster.

It is well known that relaxation phenomena can be represented by an empirical KWW law, with the exponent $\beta$ lying between 0 and $1 . \beta=1$ corresponds to a Debye-type relaxation process where molecules can relax independent of each other. There has been a great deal of discussion about whether exponent $\beta$ has any physical significance at all, as it is central in the coupling model, ${ }^{40,41}$ or if it is a purely phenomenological parameter. ${ }^{42}$ For $\beta<1$, relaxation of a macroscopic quantity can be interpreted either in terms of a distribution of relaxation times, which represents the relaxations in different static or dynamically fluctuating local environments in the system ( $\beta$ being a measure of the width of this distribution), or, in terms of a cooperative nonexponential motion due to intermolecular interactions (with $\beta$ being an indicator of the degree of cooperativity). This latter variant is the most prevalent, consistent with experimental results. A direct link between the change of the KWW exponent and the number of molecules that participate in a cooperative rearrangement, as a function of temperature, has been established in Refs. 43 and 44 in a variety of systems.

In Table II we give the stretched exponential fitting parameters of the $C_{\mathrm{CE} 1}^{1}(t), C_{\mathrm{CE} 2}^{1}(t)$, and $C_{\mathrm{CE} 3}^{1}(t)$ autocorrelation functions.

The complete dynamical behavior of the cluster is summarized in Figs. 12(a)-12(c), where the self-diffusion coefficients (a), the values of the $\beta$ exponent (b), and the reorientational relaxation times (c), are displayed as a function of the electric field.

In the following, there are a few observations to be made.

First, with the switching on of the field $(E=0.5$ $\times 10^{7} \mathrm{~V} / \mathrm{cm}$ ) there is an abrupt reduction, by almost four times, of the reorientational relaxation times for all three molecular axes, which acquire nearly equal values. In fact, the dynamic picture here is in accord with the structural picture derived previously, about the weakening of the molecular local environment with the application of the field. The reduction in particular of the $\tau^{\mathrm{CE} 3}$ reorientational time of the dipole axis, namely the increase of the flexibility of the tumbling motions of the individual molecules, is of great importance since it facilitates the molecular reorientation from ran- dom orientations at $E=0$, to preferential directions along the field at $E \neq 0$.

Second, the variation of the reorientational times with the field is profoundly nonmonotonic. Small values of the decay times are a rough indication of a disordered local environment. In contrast, larger values indicate the existence of a more stiff and ordered structure. For all fields but the strongest, at $10^{8} \mathrm{~V} / \mathrm{cm}$, all decay rates are faster than for the nofield case, despite the partial ordering observed in the fusedcubes phase.

Third, the variation of $\beta$ exponents for the CE1 and CE2 axes as a function of the field is quite similar. We observe an initial drop to small values at $E=1.0 \times 10^{7} \mathrm{~V} / \mathrm{cm}$ and an abrupt increase to values approaching 1 for $E$ larger than that. Note that this initial drop of $\beta$ is associated with the increase of translational diffusion [Fig. 12(a)] a fact that lends support to the scenario that the weakening of the network at the weak-field regime enhances molecular diffusion and favors the development of cooperative motions. Whenever diffusion is enhanced $\beta$ takes small values, and vice versa. This constitutes a clear example of coupled translational and orientational relaxations, where orientational quantities have a chance to relax only by a changing local environment. Such a coupling has been observed in super-

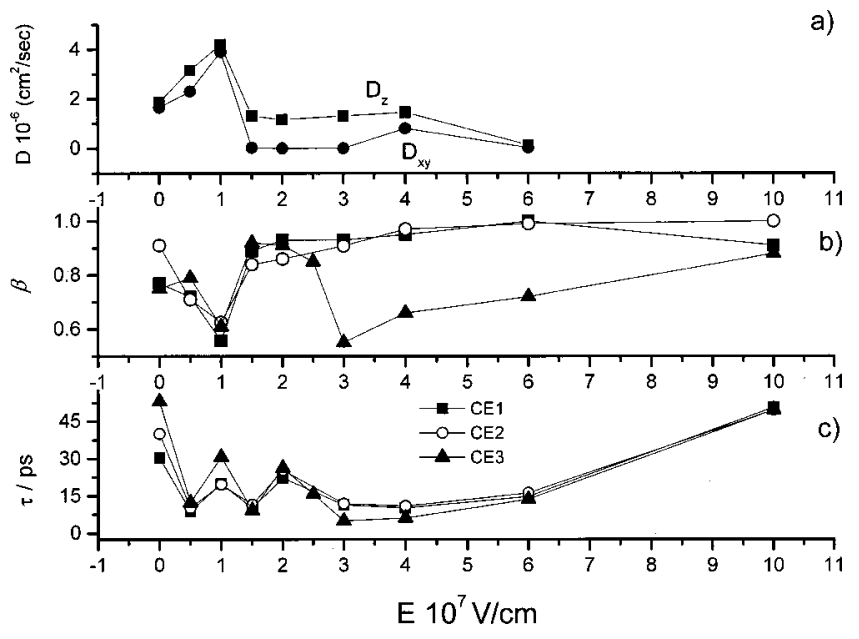

FIG. 12. (a) Longitudinal and transverse diffusion coefficients; (b) $\beta$ exponent; and (c) reorientational relaxation times as a function of the electric field. 
cooled liquid water ${ }^{45}$ and in the motion of probe molecules in polymer matrices. ${ }^{46}$ Cooperativity is a prerequisite for structural rearrangements. At $E=1.0 \times 10^{7} \mathrm{~V} / \mathrm{cm}$ the cluster, although not significantly polarized, is already considerably elongated. Its average length along the $z$ axis jumps to 41 ao at $E=1.0 \times 10^{7} \mathrm{~V} / \mathrm{cm}$, from 25.6 ao at $E=0.5 \times 10^{7} \mathrm{~V} / \mathrm{cm}$, whereas its radius in the $x-y$ plane remains unchanged at about 10ao. The transition from nearly spherical to stretched conformations with the concomitant rearrangements in the hydrogen-bonding pattern should require the cooperation of larger groups of molecules, and this effect is described by the drop in the value of the $\beta$ exponent.

As seen before [Figs. 4 and 11(b)], for $E \geqslant 1.5 \times 10^{7}$ $\mathrm{V} / \mathrm{cm}$ there is an abrupt increase in cluster polarization, which is the result of the molecular dipole axis flipping from random orientations to a distribution of angles less than 90 deg with respect to the $z$ axis. There is also a progressive ordering of the molecular dipoles along the field direction in a head-to-tail fashion. Thus, for these fields the hydrogenbonding network is developing preferentially along the $z$ axis, with the lateral molecular interactions gradually diminished. At the same time the cluster also shrinks along the perpendicular to the field directions. The resultant pattern facilitates molecular spinning rather than molecular tumbling, and the $\beta$ exponent corresponding to the reorientations of the CE1 and CE2 axes takes values approaching 1, signifying an near-independence of this kind of motion. However, this is not the case for the reorientation of the dipole axis, where cooperativity plays a very crucial role for the achievement of dipole alignment.

\section{SUMMARY AND CONCLUSIONS}

Before ending, we would like to give a summary of the major processes that are taking place in a water cluster under the influence of an external electric field. For fields in the $0-10^{8} \mathrm{~V} / \mathrm{cm}$ range, a relatively small water cluster undergoes a structural phase transition at $E=1.5 \times 10^{7} \mathrm{~V} / \mathrm{cm}$ which is related to a partial ordering of water molecules into fusedcubic structures. For fields larger than $E=3 \times 10^{7} \mathrm{~V} / \mathrm{cm}$ there is a collapse of the fused-cubic framework into more open, disordered structures that progressively become protonordered cubic forms at $E=10^{8} \mathrm{~V} / \mathrm{cm}$. Although dipole flipping takes the characteristics of a phase transition, the transition to the cubic quasicrystalline phase, which is also observed in the liquid, occurs in a continuous manner.

Observation of the $g_{\mathrm{OO}}(R)$ RDFs, of the evolution of the oxygen atom coordination number, and of the distribution of the angles between the dipole moment vectors and the field direction as a function of the external field, leaves no doubt that the resulting configurations can be classified into three distinct, well-separated groups, each of them sharing similar structural characteristics.

Apart from structural transformations, we have also examined the dynamic behavior of the cluster at the different field regimes, in terms of diffusion coefficients and single molecule reorientational times.

In the weak-field regime, a clear synergy between translational and rotational diffusion is observed. With field increase, there is an increase in diffusion both in the transverse and longitudinal directions, a reduction of the orientational relaxation times, and an increase in cooperativity of tumbling and spinning molecular motions, which in this way facilitates the dipole axis flipping process from random directions to directions closer to the $z$ axis. Together, these effects all point in the same direction, namely the weakening of the local tetrahedral structure in the cluster and the loosening of the cage of the near neighbors. In fact, a net increase in cluster volume has been calculated.

In the intermediate-field regime, the appearance of a partially ordered phase results in a sharp decrease of selfdiffusion coefficients. The reorientational times, however, remain small compared to the $E=0$ case, probably due to the nonrigidity of the nanotube conformations. The cubes do not stay very stable, since they are destroyed and reformed several times during the simulation time.

Finally, in the strong-field regime, the gradual transition to another ordered phase, at $E=10^{8} \mathrm{~V} / \mathrm{cm}$, is reflected on the $D_{z}$ falloff to smaller values. The same is observed in reorientational decay rates as well. Regarding the $\beta$ exponent, we observe a continuing loss of cooperativity in the reorientations of the CE1 and CE2 axes (spinning motions), but an abrupt increase in the cooperativity of the CE3 axis (tumbling motions). This lack of cooperativity in the molecular spins is attributed to the severe impairment of the molecular neighborhood in directions transverse to the field. For $E \geqslant 3$ $\times 10^{7} \mathrm{~V} / \mathrm{cm}$, all molecules are transferred at a larger distance in the $x-y$ plane and the meaning of a lateral hydrogen bond is lost. In contrast, the cooperativity of the tumbling motions is strengthened in order to facilitate the transition to the proton-ordered cubic phase $\left(E=10^{8} \mathrm{~V} / \mathrm{cm}\right)$.

As for our main conclusion, an external electric field applied to a water cluster can induce significant structural and dynamic changes in a nonmonotonic way. As is evident, all of these changes can have a profound effect on the reactivity and solvation capacity of the cluster. The richness of phenomena observed makes this system worth a closer look. Systematic examination of the temperature dependence of the field-induced phase transitions is the scope of a work in progress.

\section{ACKNOWLEDGMENTS}

Financial support by a grant from INTAS (No. 9901162) is gratefully acknowledged. One of the authors (A.V.) gratefully acknowledges Professor Wolffram Schröer for stimulating and helpful discussions.

\footnotetext{
${ }^{1}$ M. R. Reddy and M. Berkowitz, J. Chem. Phys. 87, 6682 (1987).

${ }^{2}$ J. S. Tse and M. L. Klein, J. Phys. Chem. 92, 315 (1988).

${ }^{3}$ J. S. Tse, J. Chem. Phys. 96, 5482 (1992).

${ }^{4}$ J. S. Tse and M. L. Klein, Phys. Rev. Lett. 58, 1672 (1987)

${ }^{5}$ J. S. Tse and M. L. Klein, J. Chem. Phys. 92, 3992 (1990).

${ }^{6}$ Y. P. Handa, J. S. Tse, D. D. Klug, and E. Whalley, J. Chem. Phys. 94, 623 (1991).

${ }^{7}$ C. H. Cho, J. Urquidi, and G. W. Robinson, J. Chem. Phys. 111, 10171 (1999).

${ }^{8}$ A. V. Okhulkov, Y. N. Demianets, and Y. E. Gorbaty, J. Chem. Phys. 100, 1578 (1994)

${ }^{9}$ G. Jancsó, P. Bopp, and K. Heinzinger, Chem. Phys. 85, 377 (1984).

${ }^{10}$ G. T. Gao, K. J. Oh, and X. C. Zeng, J. Chem. Phys. 110, 2533 (1998)

${ }^{11}$ I. M. Svishchev and P. G. Kusalik, Phys. Rev. Lett. 73, 975 (1994).
} 
${ }^{12}$ I. M. Svishchev and T. M. Hayward, J. Chem. Phys. 111, 9034 (1999).

${ }^{13}$ I. M. Svishchev and P. G. Kusalik, J. Am. Chem. Soc. 118, 649 (1996).

${ }^{14}$ I. M. Svishchev and P. G. Kusalik, Phys. Rev. B 53, 8815 (1996).

${ }^{15}$ M. Kiselev and K. Heinzinger, J. Chem. Phys. 105, 650 (1996).

${ }^{16}$ R. O. Watts, Chem. Phys. 57, 185 (1981).

${ }^{17}$ I. C. Yeh and M. L. Berkowitz, J. Chem. Phys. 110, 7935 (1999).

${ }^{18}$ X. Xia and M. L. Berkowitz, Phys. Rev. Lett. 74, 3193 (1995).

${ }^{19}$ S. B. Zhu and G. W. Robinson, J. Chem. Phys. 94, 1403 (1991).

${ }^{20}$ S. Senapati and A. Chandra, J. Mol. Struct.: THEOCHEM 455, 1 (1998).

${ }^{21}$ D. H. Jung, J. H. Yang, and M. S. Jhon, Chem. Phys. 244, 331 (1999).

${ }^{22}$ M. Watanabe, A. Brodsky, and W. P. Reinhardt, J. Phys. Chem. 95, 4593 (1991).

${ }^{23}$ H. E. Alper and R. M. Levy, J. Phys. Chem. 94, 8401 (1990).

${ }^{24}$ S. H. Lee, J. C. Rasaiah, and J. B. Hubbard, J. Chem. Phys. 86, 2383 (1987).

${ }^{25}$ I. Borzsák and P. T. Cummings, Phys. Rev. E 56, 6279 (1997).

${ }^{26}$ K. Koga, X. C. Zeng, and H. Tanaka, Phys. Rev. Lett. 79, 5262 (1997).

${ }^{27}$ C. Hartnig, W. Witschel, and E. Spohr, J. Phys. Chem. B 102, 1241 (1998).

${ }^{28}$ S. V. Shevkunov and A. Vegiri, J. Mol. Struct.: THEOCHEM (in press).

${ }^{29}$ J. Evans and S. Murad, Mol. Phys. 34, 327 (1977).

${ }^{30}$ J. Evans, Mol. Phys. 34, 317 (1977).

${ }^{31}$ L. F. Shampine and M. K. Gordon, Computer Solutions of Ordinary Differential Equations (Freeman, San Francisco, 1975).
${ }^{32}$ W. L. Jorgensen, J. Chandrasekhar, J. D. Madura, R. W. Impey, and M. L. Klein, J. Chem. Phys. 79, 926 (1983).

${ }^{33}$ P. H. Poole, U. Essmann, F. Sciortino, and H. E. Stanley, Phys. Rev. E 48, 4605 (1993).

${ }^{34}$ N. Matubayasi, C. Wakai, and M. Nakahara, J. Chem. Phys. 110, 8000 (1999).

${ }^{35}$ A. A. Chialvo and P. T. Cummings, J. Phys. Chem. 100, 1309 (1996).

${ }^{36}$ J. Rodriguez, D. Laria, E. J. Marceca, and D. A. Estrin, J. Chem. Phys. 110, 9039 (1999).

${ }^{37}$ C. J. Tsai and K. D. Jordan, J. Phys. Chem. 97, 5208 (1993).

${ }^{38}$ K. Koga, R. D. Parra, H. Tanaka, and X. C. Zeng, J. Chem. Phys. 113, 5037 (2000).

${ }^{39}$ G. Williams and D. C. Watts, Trans. Faraday Soc. 66, 80 (1970).

${ }^{40}$ K. L. Ngai, Solid State Phys. 9, 121 (1979).

${ }^{41}$ K. Y. Tsang and K. L. Ngai, Phys. Rev. E 54, 3067 (1996); 56, 17 (1997).

${ }^{42}$ W. Götze and L. Sjögren, Rep. Prog. Phys. 55, 241 (1992).

${ }^{43}$ K. L. Ngai, J. Chem. Phys. 111, 3639 (1999).

${ }^{44}$ C. León and K. L. Ngai, J. Phys. Chem. B 103, 4045 (1999).

${ }^{45}$ L. Fabbian, F. Sciortino, and P. Tartaglia, J. Non-Cryst. Solids 235-237, 325 (1998).

${ }^{46}$ T. Inoue, M. T. R. Cicerone, and M. D. Ediger, Macromolecules 28, 3425 (1995). 